\title{
Chiral perturbation theory analysis of the baryon magnetic moments revisited
}

\author{
Loyal Durand图 and Phuoc Hal \\ Department of Physics, University of Wisconsin-Madison, \\ 1150 University Avenue, Madison, WI 53706
}

(August 16, 2021)

\begin{abstract}
We reexamine critically the chiral expansion for the baryon magnetic moments including the contributions from loops which involve intermediate octet and decuplet baryons. We find that, contrary to some claims, the nonanalytic loop contributions of orders $m_{s}^{1 / 2}$ and $m_{s} \ln m_{s}$ are of the same general size because of large coupling factors for the latter, and that the decuplet contributions are as large as the octet contributions and must be included in a consistent calculation. There is no clear evidence of the convergence of the chiral series. The adequacy of the theory will not be established until dynamical models are able to calculate the contributions from the counterterms that largely hide the loop effects in fits to the data.
\end{abstract}

13.40.Em,11.30.Rd

Typeset using REVTEX

*Electronic address:ldurand@theory2.physics.wisc.edu

$\dagger$ Electronic address: phuoc@theory1.physics.wisc.edu 


\section{INTRODUCTION}

Chiral perturbation theory (ChPT) has been a very useful approach to the theory of the low momentum processes involving mesons and baryons, and has been used in various attempts to explain the baryon magnetic moments. All the moments in the baryon octet except that of the $\Sigma^{0}$ have been measured. The $\Sigma^{0} \Lambda$ transition moment $\mu_{\Sigma \Lambda}$ is also known. At the SU(3)-symmetric tree level [1], ChPT parameterizes these eight measured quantities terms of the two parameters $\mu_{D}$ and $\mu_{F}$ in the effective Lagrangian

$$
\mathcal{L}=\frac{e}{4 m_{N}}\left(\mu_{D} \operatorname{Tr} \bar{B} \sigma_{\mu \nu} F^{\mu \nu}\{Q, B\}+\mu_{F} \operatorname{Tr} \bar{B} \sigma_{\mu \nu} F^{\mu \nu}[Q, B]\right)
$$

and is able to fit them with an average error of about 0.24 nuclear magnetons $\mu_{\mathrm{N}}$. In this expression, $B$ is the usual representation for the baryon-octet in flavor-space, $Q=$ $\operatorname{diag}(2,-1,-1) / 3$ is the quark matrix, $m_{N}$ is the nucleon mass, and $F^{\mu \nu}$ is the electromagnetic field.

The study of the baryon moments beyond the tree level has a long history which can be found in [2, 3, 4, 5, 6, 6, 8, 9, 9, [10, 11]. The approaches and conclusions have varied. Caldi and Pagels [2] pointed out that the loop corrections lead to uniquely defined "nonanalytic corrections" to the moments proportional to the square root and logarithm of the symmetry breaking parameter $m_{\mathrm{s}}$ of ChPT. In contrast, the analytic corrections involving integer powers of $m_{\mathrm{s}}$ are cutoff dependent in perturbation theory. The contributions left after the divergences are subtracted are not uniquely defined in the absence of further dynamical information, and mix with the contributions of higher chiral couplings. Since these contributions are expected to be small, they have generally been ignored. Caldi and Pagels [2] calculated what were supposed to be the leading corrections to the baryon moments, those of order $m_{\mathrm{s}}^{1 / 2}$, and found that they are as large as the tree-level moments and fail to improve the fit to experiment. These terms were recalculated by Gasser et al. [3], and the logarithmic terms of type $m_{\mathrm{s}} \ln m_{\mathrm{s}}$ were analyzed by Krause [4], but without detailed analyses of the moment problem.

The problem has been reexamined in recent years. Jenkins et al. [5] used heavy baryon chiral perturbation theory (HBChPT) [12] to calculate the one-loop corrections to the octet baryon moments allowing both octet and decuplet intermediate states. Their formal analysis included the nonanalytic corrections of types $m_{\mathrm{s}}^{1 / 2}$ and $m_{\mathrm{s}} \ln m_{\mathrm{s}}$ and all the counterterms allowed to that order. However, they assumed in fitting the moment data that the logarithmic corrections and the counterterms were small compared to the terms proportional to $m_{\mathrm{s}}^{1 / 2}$, and included only the last in their analysis. The resulting fit to the data was much worse than the tree-level fit if they used tree-level values for the axial coupling constants, but better if they used the axial coupling constants extracted from one-loop fits [14 to other data, $F=0.4, D=0.61$, and $\mathcal{C}=-1.2$, values about $20 \%$ smaller than those derived at tree level. This sensitivity leaves the significance of the loop corrections unclear. In contrast, Meissner and Steininger [10] considered all octet terms (analytic and nonanalytic) which are of order $q^{4}$ or less in ChPT. They did not include the intermediate decuplet states directly, but argued that some decuplet contributions are included because of the way they determined the couplings. With the counterterms included, they could fit the seven well measured octet moments precisely, and predicted a $\Sigma \Lambda$ transition moment of $\left(1.40 \pm 0.01 \mu_{\mathrm{N}}\right)$ to be 
compared with the experimental value, $\mu_{\Sigma \Lambda}=(1.610 \pm 0.080) \mu_{\mathrm{N}}$. Their principal conclusion was that the chiral expansion appeared to be converging rather well.

The problem has also been studied using the $1 / N_{\mathrm{c}}$ expansion by a number of authors [6,7,8,9] who showed that the $m_{\mathrm{s}}^{1 / 2}$ terms are leading order in the expansion, and obtained a number of interesting sum rules for the moments. Dai et al. [9] again kept only the leading nonanalytic corrections proportional to $m_{\mathrm{s}}^{1 / 2}$ and the counterterms in their analysis of the moment data, and obtained a good overall fit, but with seven parameters. Finally, Bos et al. [11] considered the moments from the point of view of flavor $\mathrm{SU}(3)$ breaking in the baryon octet using a chiral counting and treatment of meson masses different from that usually used and again obtained a successful parametrization using seven parameters to describe eight measured moments.

\section{PREDICTIONS OF THE CHIRAL EXPANSION}

Thus far, the numerically successful approaches to the baryon moments have all used seven parameters to fit eight experimental data. Two of these parameters are the tree-level moment parameters $\mu_{D} \equiv a_{1}$ and $\mu_{F} \equiv a_{2}$. The remaining five are the coefficients $a_{3}, \ldots, a_{7}$ of the $\mathrm{SU}(3)$ symmetry breaking counterterms defined as 1

$$
\begin{aligned}
\mathcal{L}_{c t}= & \frac{e}{4 m_{N}} F^{\mu \nu}\left(a_{3} \operatorname{Tr} \bar{B}\left[\left[Q, \sigma_{\mu \nu} B\right], \mathcal{M}\right]+a_{4} \operatorname{Tr} \bar{B}\left\{\left[Q, \sigma_{\mu \nu} B\right], \mathcal{M}\right\}\right. \\
& +a_{5} \operatorname{Tr} \bar{B}\left[\left\{Q, \sigma_{\mu \nu} B\right\}, \mathcal{M}\right]+a_{6} \operatorname{Tr} \bar{B}\left\{\left\{Q, \sigma_{\mu \nu} B\right\}, \mathcal{M}\right\} \\
& \left.+a_{7} \operatorname{Tr} \bar{B} \sigma_{\mu \nu} B \operatorname{Tr} \mathcal{M} Q+\ldots\right) .
\end{aligned}
$$

Here $\mathcal{M}=\operatorname{diag}(0,0,1)$ is a matrix used to introduce $\mathrm{SU}(3)$ breaking introduced by the strange quark mass. A factor $m_{\mathrm{s}}$ has been absorbed in the coefficients of the Lagrangian. There are two terms with the same structures as those in the tree level moment operator (1) hidden in the ellipsis .

Given the large number of parameters used in the fits, it is natural to ask if the loop corrections matter, and if one can show that the uniquely extractable nonanalytic corrections actually improve the tree-level fit to the baryon moments. To answer these questions and investigate the expected cancellations and the convergence of the chiral expansion, we have redone the calculations of Jenkins et al. [5], corrected several errors in the published work, a and reanalyzed the problem of fitting the measured moments from a different point of view. We have followed Jenkins et al. in retaining only the nonanalytic contributions of types $m_{\mathrm{s}}^{1 / 2}$ and $m_{\mathrm{s}} \ln m_{\mathrm{s}}$ in our analysis, but including the effects of the octet-decuplet mass splitting throughout. The graphs which produce these contributions are shown in Figs. 1 and 2 , respectively.

\footnotetext{
${ }^{1}$ We follow the notation used in [5] except for a rearrangement of the counterterms following Bos et al. [11.

${ }^{2}$ There is a factor $(-5)$ missing in a contribution from the graph $2 \mathrm{c}$ and a factor $1 / 2$ missing from the decuplet magnetic moment operator. Other corrections are noted in the erratum to [5].
} 
The octet baryon moments are given in the one-loop approximation by

$$
\mu_{i}=\mu_{i}^{(0)}+\mu_{i}^{(1 / 2)}+\mu_{i}^{(3 / 2)},
$$

where $\mu_{i}^{(0)}$ is the tree-level moment, $\mu_{i}^{(1 / 2)}$ contains the contributions from loops that involve only intermediate spin- $1 / 2$ octet baryons, and $\mu_{i}^{(3 / 2)}$ contains the loop contributions that involve the spin-3/2 decuplet baryons,

$$
\begin{aligned}
\mu_{i}^{(0)}= & \alpha_{i}, \\
\mu_{i}^{(1 / 2)}= & \sum_{X=\pi, K} \frac{m_{N} M_{X}}{8 \pi f^{2}} \beta_{i}^{(X)}+ \\
& \sum_{X=\pi, K, \eta} \frac{1}{32 \pi^{2} f^{2}}\left(\gamma_{i}^{(X)}-2 \lambda_{i}^{(X)} \alpha_{i}\right) M_{X}^{2} \ln M_{X}^{2}, \\
\mu_{i}^{(3 / 2)}= & \sum_{X=\pi, K} \frac{m_{N}}{8 \pi f^{2}} F\left(M_{X}, \delta, \mu\right) \beta_{i}^{(X)}+ \\
& \sum_{X=\pi, K, \eta} \frac{1}{32 \pi^{2} f^{2}}\left[\left(\tilde{\gamma}_{i}^{(X)}-2 \tilde{\lambda}_{i}^{(X)} \alpha_{i}\right) L_{(3 / 2)}\left(M_{X}, \delta, \mu\right)+\hat{\gamma}_{i}^{(X)} L_{(3 / 2)}^{\prime}\left(M_{X}, \delta, \mu\right)\right] .
\end{aligned}
$$

The functions $F, L$, and $G$ are defined as

$$
\begin{aligned}
\pi F(M, \delta, \mu)= & -\delta \ln \frac{M^{2}}{\mu^{2}}+\left\{\begin{array}{ll}
2 \sqrt{M^{2}-\delta^{2}}\left[\pi / 2-\arctan \left(\delta / \sqrt{M^{2}-\delta^{2}}\right)\right], & M \geq \delta, \\
-2 \sqrt{\delta^{2}-M^{2}} \ln \left[\left(\delta+\sqrt{\delta^{2}-M^{2}}\right) / M\right], & M<\delta,
\end{array},\right. \\
L_{(3 / 2)}(M, \delta, \mu)= & M^{2} \ln \frac{M^{2}}{\mu^{2}}+2 \pi \delta F(M, \delta, \mu), \\
L_{(3 / 2)}^{\prime}(M, \delta, \mu)= & M^{2} \ln \frac{M^{2}}{\mu^{2}}+\frac{2 \pi}{3 \delta} G(M, \delta, \mu), \\
\pi G(M, \delta, \mu)= & -\delta^{3} \ln \frac{M^{2}}{\mu^{2}}+\pi M^{3} \\
& + \begin{cases}2\left(M^{2}-\delta^{2}\right)^{3 / 2}\left[\pi / 2-\arctan \left(\delta / \sqrt{M^{2}-\delta^{2}}\right)\right], & M \geq \delta, \\
-2\left(\delta^{2}-M^{2}\right)^{3 / 2} \ln \left[\left(\delta+\sqrt{\delta^{2}-M^{2}}\right) / M\right], & M<\delta,\end{cases}
\end{aligned}
$$

The coupling coefficients $\alpha_{i}, \beta_{i}, \beta_{i}^{\prime}, \lambda_{i}+\tilde{\lambda}_{i}, \gamma_{i}+\tilde{\gamma}_{i}+\hat{\gamma}_{i}$ are identical to those in [5] where $\lambda_{i}+\tilde{\lambda}_{i}$ is denoted by $\bar{\lambda}_{i}$ and $\gamma_{i}+\tilde{\gamma}_{i}+\hat{\gamma}_{i}$ is denoted by $\bar{\gamma}_{i}$. The necessary corrections have been included. The counterterm contributions follow from Eq. (2) and are given in Table I.

Before examining the loop contributions in detail, we note that the seven well-measured octet moments can be fitted exactly using the five counterterm couplings $a_{3}, \ldots, a_{7}$ and the two tree-level parameters $\mu_{D} \equiv a_{1}$ and $\mu_{F} \equiv a_{2}$, even in the presence of the loop corrections. 3 To see this, we define an $8 \times 7$ matrix $X$ which has as its $i$ th column the matrix elements of

\footnotetext{
${ }^{3}$ We choose to focus on the seven baryon moments in our analysis because of the high accuracy with which they are known relative to $\mu_{\Sigma \Lambda}$. The alternative of making a weighted least-squares
} 
the corresponding operator in the physical baryon states $p, n, \Sigma^{+}, \Sigma^{-}, \Xi^{0}, \Xi^{-}, \Lambda$ and the $\Sigma^{0} \Lambda$ transition matrix element as in Table I. The most general column vector of magnetic moments consistent with this chiral structure is then of the form

$$
\mu=X a
$$

where $a$ is a seven-component column vector of coefficients. The $7 \times 7$ matrix $\hat{X}$ obtained by omitting the $\Sigma^{0} \Lambda$ row in $X$ is invertible, so coefficients $a_{i}$ can be found which give exact fits to either the seven well-measured baryon moments, $a=\hat{X}^{-1} \hat{\mu}_{\text {meas }}$, or to the difference between the measured moments and the loop corrections in this sector, $a^{\prime}=\hat{X}^{-1}\left(\hat{\mu}_{\text {meas }}-\hat{\mu}_{\text {loop }}\right)$. In the second case, the moments are again reproduced exactly when the loop corrections are added in. The only remaining constraint on the theory is that provided by the $\Sigma^{0} \Lambda$ transition moment.

In the absence of loop corrections, $\mu_{\Sigma \Lambda}$ is determined entirely by the seven coefficients $a$ through the product $X a$ with the full matrix $X$, or, equivalently, through the Okubo sum rule [13]

$$
4 \sqrt{3} \mu_{\Sigma \Lambda}=6 \mu_{\Lambda}+\mu_{\Sigma^{-}}+\mu_{\Sigma^{+}}-4 \mu_{n}-4 \mu_{\Xi^{0}}
$$

The origin of the sum rule can be seen rather simply in the present context. We can extend the matrix $X$ to an $8 \times 8$ matrix $\hat{X}$ by adding a final column with zero entries without affecting the seven baryon moments. That is, $\mu=\hat{X} a$ with the same coefficients $a$ as determined above. However, the extended matrix has one zero eigenvalue. The sum rule is just the inner product of the left null eigenvector $\tilde{x}_{0}$ with $\mu=X a, \tilde{x}_{0} \mu=\tilde{x}_{0} \hat{X} a=0$. In particular, any set of moments which can be described by the initial seven-component chiral structure must satisfy the sum rule exactly.

The sum rule is, in fact, satisfied rather accurately by the measured moments. The predicted value of $\mu_{\Sigma \Lambda}$ is $\mu_{\Sigma \Lambda}=(1.483 \pm 0.012) \mu_{\mathrm{N}}$, to be compared with the measured value $\left.\left|\mu_{\Sigma \Lambda}\right|=1.610 \pm 0.080\right) \mu_{\mathrm{N}}$. The discrepancy is at the level of $1.5 \sigma$ or $8 \%$ of the measured transition moment.

The situation is potentially different when loop corrections are included. While the $m_{\mathrm{s}}^{1 / 2}$ corrections can be described using only the chiral structures considered so far and therefore satisfy the Okubo sum rule exactly [0], that is not the case for the corrections proportional to $m_{\mathrm{s}} \ln m_{\mathrm{s}}$, a point misstated by those authors. The logarithmic corrections involve sums of terms of the form $M_{i}^{2} \ln \left(M_{i} / \lambda\right)$ multiplied by coupling coefficients. If the mass dependence of the logarithms could be neglected, the sum rule would hold exactly for external meson masses that satisfy the Gell-Mann-Okubo mass formula. As a result, the chiral cutoff $\lambda$ drops out of the sum rule, and the violation depends only on the logarithm of the mass ratio, $\ln \left(M_{K} / M_{\pi}\right)$, hence would vanish for equal meson masses. However, the sum rule is

fit to all eight measured quantities leads to essentially identical results because of the low weight accorded $\mu_{\Sigma \Lambda}$ in the fit. The unweighted fits used in [9, 11, 10 distribute the uncertainty in $\mu_{\Sigma \Lambda}$ over all the moments without changing the one test of the theory, as will be seen below. We believe that the present procedure is clearer. 
violated for $M_{K} \neq M_{\pi}$, and the logarithmic loop corrections change the prediction for $\mu_{\Sigma \Lambda}$ and introduce new structure in the $8 \times 8$ moment space.

To investigate this point in more detail, we have constructed exact fits to the seven octet moments using the results for the nonanalytic parts of the loop corrections given in Eqs. (3 $3[10)$ using the corrected coupling coefficients from [5], and have then calculated $\mu_{\Sigma \Lambda}$. For illustration, we will present the results for the two cases considered in [5]: (a) $F=0.4$, $D=0.61$, and $\mathcal{C}=-1.2$, the one-loop axial vector couplings derived in [14]; and (b) $F=0.5$, $D=0.75$, and $\mathcal{C}=-1.5$, the tree-level couplings. In both cases, we take the decuplet-octet mass difference as $\delta=0.3 \mathrm{GeV}$, and use $f_{\pi}=0.093 \mathrm{GeV}, f_{K}=f_{\eta}=1.2 f_{\pi}, \mu_{T}=-7.7$, $\mu_{C}=1.94$, and a chiral cutoff $\lambda=1 \mathrm{GeV}$ as in that reference.

Although the sum rule is violated analytically, we find that the violation is remarkably small numerically. In particular, $\mu_{\Sigma \Lambda}$ predicted to be $1.508 \mu_{\mathrm{N}}$ for the parameters in case (a), rather the $1.483 \mu_{\mathrm{N}}$ as required by the sum rule. The difference is quite small compared to the experimental uncertainty in $\mu_{\Sigma \Lambda}$. The results in case (b) are similar, with a predicted transition moment $\mu_{\Sigma \Lambda}=1.534$. The chiral parameters for these two fits are given in Table II.

It is evident from Table II that the fitted parameters $\mu_{F}$ and $\mu_{D}$ are consistent with $\mathrm{SU}(6): \mu_{D} / \mu_{F} \sim 3 / 2$. That is expected in the usual quark model picture. The axial coupling constants $F, D$, and $\mathcal{C}$ also satisfy the $\mathrm{SU}(6)$ relations.

The result that loop corrections lead to only small violations of the Okubo sum rule appears to be quite robust. In particular, the changes in the loop corrections associated with different treatments of the meson-baryon couplings are largely absorbed by changes in the fitted counterterm parameters. We conclude that the theory with all the counterterms present is not usefully predictive at the present time. The results do not, for example, distinguish in a useful way between the standard chiral approach with the nonanalytic contributions singled out, the approach of Meissner and Steininger [10 which includes analytic as well as nonanalytic terms, or the approach of Bos et al. [11] which allows only corrections that break SU(3) symmetry linearly, fits the data with just the seven parameters above, and satisfies the sum rule exactly.

\section{ANALYSIS OF THE LOOP CORRECTIONS}

The insensitivity of the overall fits to loop effects raises the question of whether there is any evidence that these effects are actually important. The difficulty is that the counterterm contributions are not calculable in the present theory, yet are essential for getting good overall fits to the data. A breakdown of the detailed contributions to the moments for case (b) is given in Table III. The results for case (a) are similar.

The structure of the loop contributions is of considerable interest. We will examine this separately for the octet and decuplet cases.

\footnotetext{
${ }^{4}$ The new structure can be parametrized by a single invariant $\operatorname{Tr} \bar{B} M Q B M$ of order $m_{s}^{2}$ which affects only the $\Lambda$ moment.
} 


\section{A. Octet baryon contributions}

As shown in Table III, the contributions of type $m_{s}^{1 / 2}$ from the intermediate octet states are quite large, comparable to the tree-level contributions. The $m_{\mathrm{s}} \ln m_{\mathrm{s}}$ corrections have the same general magnitude, but opposite signs. As a result there are large cancellations between these two types of nonanalytic contributions. This contradicts the arguments of chiral perturbation theory [2,5] and the $1 / N_{\mathrm{c}}$ expansion [6,7,8,9] that the $m_{\mathrm{s}} \ln m_{\mathrm{s}}$ corrections should be small, of the same order of magnitude as the counterterms and ignorable relative to the $m_{\mathrm{s}}^{1 / 2}$ contributions. In fact, the smallness of the characteristic ratio [5]

$$
\left(\frac{M_{X}^{2}}{32 \pi^{2} f_{X}^{2}} \ln \frac{M_{X}^{2}}{\lambda^{2}}\right)\left(\frac{M_{X} m_{N}}{8 \pi f_{X}^{2}}\right)^{-1}=\frac{M_{X}}{4 \pi m_{N}} \ln \frac{M_{X}^{2}}{\lambda^{2}}
$$

of the two types of nonanalytic terms, approximately $-1 / 22$ and $-1 / 17$ for $X$ a pion or a kaon, is offset by very large ratios of the effective couplings, e.g., 16.1 and 12.7 for the pion and kaon contributions to the proton moment in case (b), and -28.6 and 9.9 for the $\Xi^{0}$, and the large cancellations ensue. There is no indication in this sector that the chiral perturbation series is converging.

\section{B. Decuplet contributions}

The contributions from intermediate decuplet states introduce a new complication, namely the dependence of the results on the octet-decuplet mass difference $\delta$. The results are very sensitive to $\delta$, which is a QCD parameter rather than a chiral parameter.5 Meissner and Steininger [10] argue that the decuplet contributions should be included in the chiral momentum expansion, expand in powers of $q / \delta$, and evaluate their couplings to take the resulting effects into account. Unfortunately, this argument requires that $\delta$ be large on the scale of the momenta in the loop integrals. It is not, with $M_{\pi}<\delta<M_{K}$ for typical mass splittings $\delta \approx 300 \mathrm{MeV}$, and the heavy decuplet argument fails. We conclude that the decuplet states must be regarded as light, on the same mass scale as the octet states, to obtain a consistent picture. Their contribution to the moments is then given by the expressions in Eqs. (3-10). The approximation in [10] becomes useful only for excited baryon multiplets, and can presumably be used to estimate those contributions.

Jenkins et al. included the effects of the mass splitting in the $m_{s}^{1 / 2}$ terms, but took $\delta=0$ in the logarithmic contributions. This introduces very large changes in the latter, but does not affect their final conclusions because the differences are largely compensated through the counterterms.

Using the value $\delta=300 \mathrm{MeV}$, we obtain the results for the decuplet contributions shown in Table III. The logarithmic contributions associated with the decuplet are large and tend to cancel the logarithmic corrections from the octet as suggested in [5], but the cancellation is far from complete and all the logarithmic terms must be included.

\footnotetext{
${ }^{5}$ E.g., $\delta$ is described in the quark model in terms of a color hyperfine splitting between the spin- $1 / 2$ octet and the spin-3/2 decuplet states.
} 
If we look at Table III from the point of view of a loop expansion, we see that the total contributions from the octet loops in Figs. 1 and 2 are considerably smaller than the individual $m_{s}^{1 / 2}$ and $m_{s} \ln m_{s}$ terms because of the cancellations. This suggests that a loop expansion may converge more rapidly than the chiral expansion. However, the two types of terms tend to add rather than cancel for the decuplet, and turn out to dominate the final loop corrections. The counterterms are also quite significant. We see no convincing evidence that the chiral expansion with only the nonanalytic terms retained is under control. Our attempts to determine whether or not the loop corrections improve the tree-level fit to the moments are also inconclusive. We have refit the data omitting the counterterms, but with the loop corrections included and $\mu_{D}$ and $\mu_{F}$ readjusted. The quality of the fits is essentially unchanged with respect to tree level if all loop contributions are included. Jenkins et al. find that the fits can be improved by omitting the logarithmic corrections and readjusting $F$ and $D$ in addition to the somewhat small values quoted for case (a), but this procedure is not justified by the relative sizes of the terms retained and omitted (see Table III).

\section{Conclusions}

The foregoing results suggest that the loop expansion may give a more useful approach to the calculation of the baryon moments than the chiral and $1 / N_{\mathrm{c}}$ expansions. In particular, the results obtained from the different diagrams in Figs. 1 and 2 are all of comparable size when combined with the relevant coupling factors and must be treated together to get meaningful results. However, loop effects are largely wiped out in the fits to the data when all the counterterms are treated as free parameters. Neither the chiral nor loop expansion is usefully predictive in this setting given the experimental and theoretical uncertainties. It is not possible, for example, to distinguish between the standard chiral expansion and the alternative approach proposed by Bos et al. [11]. We conclude that it will be necessary to develop a dynamical theory in which the baryons are treated as composite and the counterterms become calculable before the moment problem can be regarded as solved.

\section{ACKNOWLEDGMENTS}

The authors would like to thank Profs. Elizabeth Jenkins, Roxanne Springer, and Ulf-G. Meissner for useful correspondence about their published results. This work was supported in part by the U.S. Department of Energy under Contract No. DE-FG02-95ER40896, and in

part by the University of Wisconsin Graduate School with funds granted by the Wisconsin Alumni Research Foundation. 


\section{REFERENCES}

[1] S. Coleman and S.L. Glashow, Phys. Rev. Lett. 6, 423 (1961).

[2] D.G. Caldi and H. Pagels, Phys. Rev. D 10, 3739 (1974).

[3] J. Gasser, M. Sainio, and A. Svarc, Nucl. Phys. B307, 779 (1988).

[4] A. Krause, Helv. Phys. Acta 63, 3 (1990).

[5] E. Jenkins, M. Luke, A.V. Manohar and M. Savage, Phys. Lett. B 302, 482 (1993); (E) ibid. 388, 866 (1996).

[6] R. Dashen, E. Jenkins, and A.V. Manohar, Phys. Rev. D 49, 4713 (1994).

[7] E. Jenkins and A.V. Manohar, Phys. Lett. B 335, 452 (1994).

[8] M.A. Luty, J. March-Russell, and M. White, Phys. Rev. D 51, 2332 (1995).

[9] J. Dai, R. Dashen, E. Jenkins, and A.V. Manohar, Phys. Rev. D 53, 273 (1996).

[10] Ulf-G. Meißner, S. Steininger, Nucl. Phys. B499, 349 (1997).

[11] J.W. Bos, D. Chang, S.C. Lee, Y.C. Lin and H.H. Shih, Chin. J. Phys. (Taipei) 35, 150 (1997).

[12] H. Georgi, Phys. Lett. B 240, 447 (1990); E. Jenkins and A.V. Manohar, Phys. Lett. B 255, 558 (1991); UCSD/PTH 91-30.

[13] S. Okubo, Phys. Lett. 4, 14 (1963).

[14] E. Jenkins and A.V. Manohar, in Proc. Workshop on Effective Field Theories of the Standard Model, edited by U. Meissner (World Scientific, Singapore, 1992); M.N. Butler, M.J. Savage, and R.P. Springer, Nucl. Phys. B399, 69 (1993). 


\section{FIGURES}

FIG. 1. The diagrams which give the non-analytic $m_{\mathrm{S}}^{1 / 2}$ corrections to the baryon magnetic moments. Dashed lines denotes mesons; single and double solid lines denote octet and decuplet baryons, respectively.

FIG. 2. The diagrams which give the non-analytic $m_{\mathrm{s}} \ln m_{\mathrm{s}}$ corrections to the baryon magnetic moments. 


\section{TABLES}

TABLE I. Table of the coefficients of the counterterm contributions to the baryon magnetic moments. This corresponds to the matrix $X$ defined in the text. The notation is as follows: $a_{1}=\mu_{D}, a_{2}=\mu_{F}$, Eq. (1), while $a_{3}, \ldots, a_{7}$ are the parameters are defined in Eq. (2). The $\Sigma^{0}$ moment is not included, but is given by the $\mathrm{SU}(2)$ relation $\mu_{\Sigma^{0}}=\left(\mu_{\Sigma^{+}}+\mu_{\Sigma^{-}}\right) / 2$.

\begin{tabular}{cccccccc}
\hline \hline Baryon & $a_{1}$ & $a_{2}$ & $a_{3}$ & $a_{4}$ & $a_{5}$ & $a_{6}$ & $a_{7}$ \\
\hline$p$ & $\frac{1}{3}$ & 1 & 1 & 1 & $\frac{1}{3}$ & $\frac{1}{3}$ & $-\frac{1}{3}$ \\
$n$ & $-\frac{2}{3}$ & 0 & 0 & 0 & $-\frac{2}{3}$ & $-\frac{2}{3}$ & $-\frac{1}{3}$ \\
$\Sigma^{+}$ & $\frac{1}{3}$ & 1 & 0 & 0 & 0 & 0 & $-\frac{1}{3}$ \\
$\Sigma^{-}$ & $\frac{1}{3}$ & -1 & 0 & 0 & 0 & 0 & $-\frac{1}{3}$ \\
$\Xi^{0}$ & $-\frac{2}{3}$ & 0 & 0 & 0 & $\frac{2}{3}$ & $-\frac{2}{3}$ & $-\frac{1}{3}$ \\
$\Xi^{-}$ & $\frac{1}{3}$ & -1 & 1 & -1 & $-\frac{1}{3}$ & $\frac{1}{3}$ & $-\frac{1}{3}$ \\
$\Lambda$ & $-\frac{1}{3}$ & 0 & 0 & 0 & 0 & $-\frac{8}{9}$ & $-\frac{1}{3}$ \\
$\Sigma^{0} \Lambda$ & $\frac{1}{\sqrt{3}}$ & 0 & 0 & 0 & 0 & 0 & 0 \\
\hline \hline
\end{tabular}

TABLE II. The best fit parameters obtained by fitting the seven measured octet magnetic moments exactly. The parameters are given in units of $\mu_{N}$. The $\Sigma \Lambda$ transition moment is predicted to be $\mu_{\Sigma \Lambda}=1.508 \mu_{\mathrm{N}}$ in case (a), $1.534 \mu_{\mathrm{N}}$ in case (b), and (1.483 \pm 0.012$) \mu_{\mathrm{N}}$ by the Okubo relation, case (c).

\begin{tabular}{llllllrr}
\hline \hline Case & $a_{1}$ & $a_{2}$ & $a_{3}$ & $a_{4}$ & $a_{5}$ & \multicolumn{1}{c}{$a_{6}$} & \multicolumn{1}{c}{$a_{7}$} \\
\hline (a) & 3.183 & 1.992 & 0.269 & -0.215 & 0.534 & 0.224 & -0.392 \\
(b) & 3.946 & 2.353 & -0.001 & -0.172 & 0.569 & 0.694 & -1.165 \\
(c) & 2.568 & 1.809 & 0.591 & -0.253 & 0.497 & -0.506 & 0.621 \\
\hline \hline
\end{tabular}

TABLE III. Detailed breakdown of the contributions of the loop integrals and the counterterms to the fitted magnetic moments of the octet baryons for the parameters of case (b), Table II. The first seven moments were used as input, and are fitted exactly. The only prediction of the theory is $\mu_{\Sigma \Lambda}$.

\begin{tabular}{crrrrrrrr}
\hline \hline$\mu_{B}$ & \multicolumn{1}{c}{$\mu_{D}, \mu_{F}$} & \multicolumn{1}{c}{$m_{s}^{1 / 2}(N)$} & \multicolumn{1}{c}{$m_{s}^{1 / 2}(\Delta)$} & $\ln m_{s}(N)$ & $\ln m_{s}(\Delta)$ & \multicolumn{1}{c}{ Loops } & \multicolumn{1}{c}{$\mathrm{CT}$} & \multicolumn{1}{c}{$\mu_{B}$} \\
\hline $\mathrm{p}$ & 3.668 & -2.227 & -0.162 & 2.148 & -1.271 & -1.512 & 0.636 & 2.793 \\
$\mathrm{n}$ & -2.631 & 0.832 & 0.573 & -1.491 & 1.258 & 1.172 & -0.454 & -1.913 \\
$\Sigma^{+}$ & 3.668 & -2.843 & -0.474 & 2.829 & -1.111 & -1.598 & 0.388 & 2.458 \\
$\Sigma^{-}$ & -1.037 & 0.611 & -0.349 & -0.804 & 0.031 & -0.511 & 0.388 & -1.160 \\
$\Sigma^{0}$ & 1.315 & -1.116 & -0.411 & 1.012 & -0.540 & -1.055 & 0.388 & 0.649 \\
$\Lambda$ & -1.315 & 1.116 & 0.411 & -1.211 & 0.614 & 0.931 & -0.228 & -0.613 \\
$\Xi^{0}$ & -2.631 & 2.288 & 0.698 & -2.650 & 0.739 & 1.075 & 0.306 & -1.250 \\
$\Xi^{-}$ & -1.037 & 1.339 & -0.286 & -1.324 & 0.057 & -0.215 & 0.601 & -0.651 \\
$\Sigma^{0} \Lambda$ & 2.278 & -1.157 & -0.496 & 1.680 & -0.771 & -0.745 & 0.000 & 1.534 \\
\hline \hline
\end{tabular}




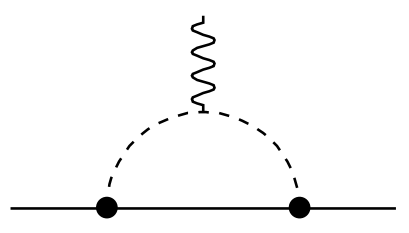

(a)

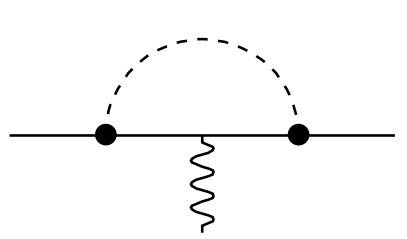

(a)

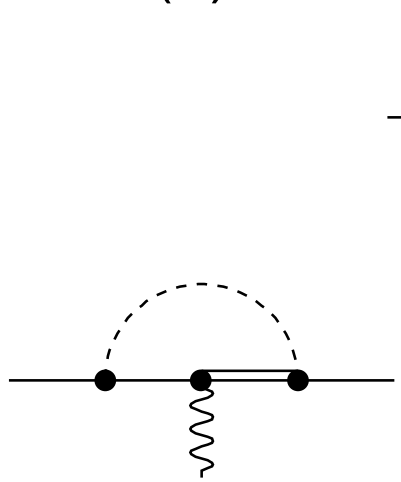

(d)

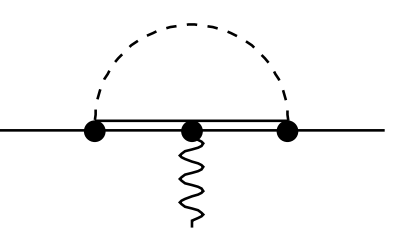

(c)

Fig. 1

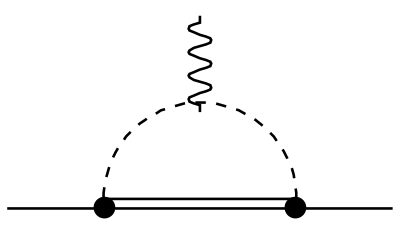

(b)

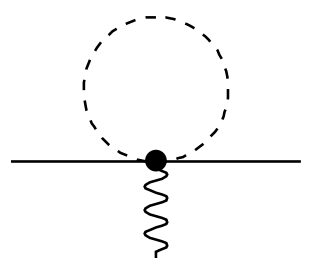

(b)

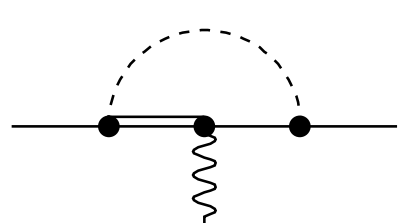

(e)

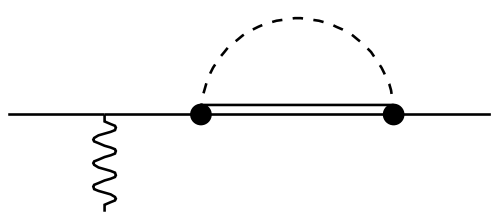

(g)

Fig. 2 